\title{
Existence results for fractional differential inclusions with Erdélyi-Kober fractional integral conditions
}

\author{
Bashir Ahmad and Sotiris K. Ntouyas
}

\begin{abstract}
In this paper, we discus the existence of solutions for RiemannLiouville fractional differential inclusions supplemented with ErdélyiKober fractional integral conditions. We apply endpoint theory, Krasnoselskii's multi-valued fixed point theorem and Wegrzyk's fixed point theorem for generalized contractions. For the illustration of our results, we include examples.
\end{abstract}

\section{Introduction}

Differential inclusions, regarded as generalization of differential equations and inequalities, are found to be of great interest and value in optimal control theory and stochastic processes [1]. Differential inclusions also help to understand dynamical systems in which the velocities are not uniquely specified by the state of the system, in spite of depending on it.

In recent years, the area of differential equations and inclusions of arbitrary order has received considerable attention and several results of wide interest can now be found in the literature on the topic, for instance, see [2]-[7] and the references cited therein.

Key Words: Riemann-Liouville fractional derivative, inclusions; Erdélyi-Kober fractional integral, endpoint theory, Krasnoselskii's multi-valued fixed point theorem; Wegrzyk's fixed point theorem.

2010 Mathematics Subject Classification: Primary 34A08, 34A12. Secondary 34A60.

Received: February, 2016.

Revised: April, 2016.

Accepted: May, 2016. 
It has been observed that much of the work dealing with fractional-order boundary problems equipped with integral boundary conditions has addressed either classical, Riemann-Liouville or Hadamard type integrals. Besides these integrals, there is another kind of integral operator, introduced by Arthur Erdélyi and Hermann Kober [8] in 1940, which is known as Erdélyi-Kober fractional integral operator. Such operators are found to be quite useful in obtaining the solutions for single, dual and triple integral equations possessing special functions of mathematical physics in their kernels. For details and applications of the Erdélyi-Kober fractional integrals, we refer the reader to a series of papers and texts $[8,9,10,11,12,13]$.

In order to enrich the work on fractional-order boundary value problems involving integral boundary conditions further, we consider a problem consisting of fractional differential inclusions and Erdélyi-Kober fractional integral boundary conditions. Precisely, we investigate the following problem:

$$
\left\{\begin{array}{l}
D^{q} x(t) \in F(t, x(t)), \quad 0<t<T, \quad 1<q \leq 2, \\
x(0)=0, \\
\alpha x(T)=\sum_{i=1}^{m} \beta_{i} \frac{\eta t^{-\eta_{i}\left(\delta_{i}+\gamma_{i}\right)}}{\Gamma\left(\delta_{i}\right)} \int_{0}^{\xi_{i}} \frac{s^{\eta_{i} \gamma_{i}+\eta_{i}-1} x(s)}{\left(t^{\eta_{i}}-s^{\eta_{i}}\right)^{1-\delta_{i}}} d s:=\sum_{i=1}^{m} \beta_{i} I_{\eta_{i}}^{\gamma_{i}, \delta_{i}} x\left(\xi_{i}\right),
\end{array}\right.
$$

where $1<q \leq 2, D^{q}$ is the standard Riemann-Liouville fractional derivative of order $q, I_{\eta_{i}}^{\gamma_{i}, \delta_{i}}$ is the Erdélyi-Kober fractional integral of order $\delta_{i}>0$ with $\eta_{i}>0$ and $\gamma_{i} \in \mathbb{R}, i=1,2, \ldots, m, F:[0, T] \times \mathbb{R} \rightarrow \mathcal{P}(\mathbb{R})$ is a multivalued map, $\mathcal{P}(\mathbb{R})$ is the family of all nonempty subsets of $\mathbb{R}$, and $\alpha, \beta_{i} \in \mathbb{R}, \xi_{i} \in(0, T)$, $i=1,2, \ldots, m$ are given constants.

In the rest of the paper, we describe some preliminary concepts related to the proposed study in Section 2, while the main existence results are established in Section 3 by applying endpoint theory, Krasnoselskii's multi-valued fixed point theorem and Wegrzyk's fixed point theorem for generalized contractions. Finally we present examples for illustration of our main results.

\section{Preliminaries}

\subsection{Basic material for fractional calculus}

In this section, we introduce some notations and definitions of fractional calculus and present preliminary results needed in our proofs later [13, 14].

Definition 2.1. The Riemann-Liouville fractional derivative of order $q>0$ 
of a continuous function $f:(0, \infty) \rightarrow \mathbb{R}$ is defined by

$$
D^{q} f(t)=\frac{1}{\Gamma(n-q)}\left(\frac{d}{d t}\right)^{n} \int_{0}^{t}(t-s)^{n-q-1} f(s) d s, \quad n-1<q<n,
$$

where $n=[q]+1,[q]$ denotes the integer part of a real number $q$. Here $\Gamma$ is the Gamma function defined by $\Gamma(q)=\int_{0}^{\infty} e^{-s} s^{q-1} d s$.

Definition 2.2. The Riemann-Liouville fractional integral of order $q>0$ of a continuous function $f:(0, \infty) \rightarrow \mathbb{R}$ is defined by

$$
J^{q} f(t)=\frac{1}{\Gamma(q)} \int_{0}^{t}(t-s)^{q-1} f(s) d s
$$

provided the integral exists.

Definition 2.3. The Erdélyi-Kober fractional integral of order $\delta>0$ with $\eta>0$ and $\gamma \in \mathbb{R}$ of a continuous function $f:(0, \infty) \rightarrow \mathbb{R}$ is defined by

$$
I_{\eta}^{\gamma, \delta} f(t)=\frac{\eta t^{-\eta(\delta+\gamma)}}{\Gamma(\delta)} \int_{0}^{t} \frac{s^{\eta \gamma+\eta-1} f(s)}{\left(t^{\eta}-s^{\eta}\right)^{1-\delta}} d s
$$

provided the right side is pointwise defined on $\mathbb{R}_{+}$.

Lemma 2.1. Let $\delta, \eta>0$ and $\gamma, q \in \mathbb{R}$. Then we have

$$
I_{\eta}^{\gamma, \delta} t^{q}=\frac{t^{q} \Gamma(\gamma+(q / \eta)+1)}{\Gamma(\gamma+(q / \eta)+\delta+1)} .
$$

Lemma 2.2. Let $1<q \leq 2, \delta_{i}, \eta_{i}>0, \alpha, \gamma_{i}, \beta_{i} \in \mathbb{R}, \xi_{i} \in(0, T), i=$ $1,2, \ldots, m$ and $h \in A C([0, T], \mathbb{R})$. Then the linear Riemann-Liouville fractional differential equation subject to the Erdélyi-Kober fractional integral boundary conditions

$$
\left\{\begin{array}{l}
D^{q} x(t)=h(t), \quad t \in(0, T), \\
x(0)=0, \quad \alpha x(T)=\sum_{i=1}^{m} \beta_{i} I_{\eta_{i}}^{\gamma_{i}, \delta_{i}} x\left(\xi_{i}\right),
\end{array}\right.
$$

is equivalent to the following integral equation

$$
x(t)=J^{q} h(t)-\frac{t^{q-1}}{\Lambda}\left(\alpha J^{q} h(T)-\sum_{i=1}^{m} \beta_{i} I_{\eta_{i}}^{\gamma_{i}, \delta_{i}} J^{q} h\left(\xi_{i}\right)\right),
$$

where

$$
\Lambda:=\alpha T^{q-1}-\sum_{i=1}^{m} \frac{\beta_{i} \xi_{i}^{q-1} \Gamma\left(\gamma_{i}+(q-1) / \eta_{i}+1\right)}{\Gamma\left(\gamma_{i}+(q-1) / \eta_{i}+\delta_{i}+1\right)} \neq 0
$$


Proof. Solving the linear fractional differential equation in (3), we get an integral equation given by

$$
x(t)=J^{q} h(t)-c_{1} t^{q-1}-c_{2} t^{q-2},
$$

where $c_{1}, c_{2} \in \mathbb{R}$. The first condition $(x(0)=0)$ of (3) implies that $c_{2}=0$. Applying the Erdélyi-Kober fractional integral operator of order $\delta_{i}>0$ with $\eta_{i}>0$ and $\gamma_{i} \in \mathbb{R}$ on (6) and using Lemma 2.1, we obtain

$$
I_{\eta_{i}}^{\gamma_{i}, \delta_{i}} x(t)=I_{\eta_{i}}^{\gamma_{i}, \delta_{i}} J^{q} h(t)-c_{1} \frac{t^{q-1} \Gamma(\gamma+(q-1) / \eta+1)}{\Gamma(\gamma+(q-1) / \eta+\delta+1)},
$$

which, together with the second condition of (3), yields

$\alpha J^{q} h(T)-c_{1} \alpha T^{q-1}=\sum_{i=1}^{m} \beta_{i} I_{\eta_{i}}^{\gamma_{i}, \delta_{i}} J^{q} h\left(\xi_{i}\right)-c_{1} \sum_{i=1}^{m} \frac{\beta_{i} \xi_{i}^{q-1} \Gamma\left(\gamma_{i}+(q-1) / \eta_{i}+1\right)}{\Gamma\left(\gamma_{i}+(q-1) / \eta_{i}+\delta_{i}+1\right)}$.

Thus

$$
c_{1}=\frac{1}{\Lambda}\left(\alpha J^{q} h(T)-\sum_{i=1}^{m} \beta_{i} I_{\eta_{i}}^{\gamma_{i}, \delta_{i}} J^{q} h\left(\xi_{i}\right)\right),
$$

where $\Lambda$ is given by (5). Substituting the values of $c_{1}$ and $c_{2}$ in (6), we obtain the desired solution (4). The converse follows by direct computation.

\subsection{Basic material for multivalued maps}

Here we outline some basic concepts of multivalued analysis $[15,16]$.

Let $\mathcal{U}:=C([0, T], \mathbb{R})$ denote the Banach space of all continuous functions from $[0, T]$ into $\mathbb{R}$ with the norm $\|x\|=\sup \{|x(t)|, t \in[0, T]\}$. Also by $L^{1}([0, T], \mathbb{R})$ we denote the space of functions $x:[0, T] \rightarrow \mathbb{R}$ such that $\|x\|_{L^{1}}=\int_{0}^{T}|x(t)| d t$.

For a normed space $(X,\|\cdot\|)$, we define $\mathcal{P}_{c l}(X)=\{Y \in \mathcal{P}(X): Y$ is closed $\}$, $\mathcal{P}_{b d}(X)=\{Y \in \mathcal{P}(X): Y$ is bounded $\}, \mathcal{P}_{c p}(X)=\{Y \in \mathcal{P}(X): Y$ is compact $\}$ $\mathcal{P}_{c l, b d}(X)=\{Y \in \mathcal{P}(X): Y$ is closed and bounded $\}$, and $\mathcal{P}_{c p, c}(X)=\{Y \in$ $\mathcal{P}(X): Y$ is compact and convex $\}$.

A multi-valued map $G: X \rightarrow \mathcal{P}(X)$ is (i) convex (closed) valued if $G(x)$ is convex (closed) for all $x \in X$; (ii) bounded on bounded sets if $G(Y)=$ $\cup_{x \in Y} G(x)$ is bounded in $X$ for all $Y \in \mathcal{P}_{b}(X)$ (i.e. $\sup _{x \in Y}\{\sup \{|y|: y \in$ $G(x)\}\}<\infty)$;(iii) is called upper semi-continuous (u.s.c.) on $X$ if for each $x_{0} \in X$, the set $G\left(x_{0}\right)$ is a nonempty closed subset of $X$, and if for each open set $N$ of $X$ containing $G\left(x_{0}\right)$, there exists an open neighborhood $\mathcal{N}_{0}$ of $x_{0}$ such that $G\left(\mathcal{N}_{0}\right) \subseteq N$; (iv) lower semi-continuous (l.s.c.) if the set 
$\{y \in X: G(y) \cap Y \neq \emptyset\}$ is open for any open set $Y$ in $X$; (v) completely continuous if $G(\mathbb{B})$ is relatively compact for every $\mathbb{B} \in \mathcal{P}_{b}(X)$.

If the multi-valued map $G$ is completely continuous with nonempty compact values, then $G$ is u.s.c. if and only if $G$ has a closed graph, that is, $x_{n} \rightarrow x_{*}, y_{n} \rightarrow y_{*}, y_{n} \in G\left(x_{n}\right)$ imply $y_{*} \in G\left(x_{*}\right)$. The multi-valued map $G$ is said to be measurable if for every $y \in X$, the function $t \longmapsto d(y, G(t))=$ $\inf \{|y-z|: z \in G(t)\}$ is measurable. The multi-valued map $G$ has a fixed point if there is $x \in X$ such that $x \in G(x)$. The fixed point set of the multivalued operator $G$ will be denoted by Fix $G$.

Let $(X, d)$ be a metric space induced from the normed space $(X ;\|\cdot\|)$. Consider $H_{d}: \mathcal{P}(X) \times \mathcal{P}(X) \rightarrow \mathbb{R} \cup\{\infty\}$ given by

$$
H_{d}(A, B)=\max \left\{\sup _{a \in A} d(a, B), \sup _{b \in B} d(A, b)\right\},
$$

where $d(A, b)=\inf _{a \in A} d(a ; b)$ and $d(a, B)=\inf _{b \in B} d(a ; b)$. Then $\left(\mathcal{P}_{c l, b}(X), H_{d}\right)$ is a metric space (see [17]).

Definition 2.4. A function $x \in A C^{2}(J, \mathbb{R})$ is called a solution of problem (1) if there exists a function $v \in L^{1}(J, \mathbb{R})$ with $v(t) \in F(t, x(t))$, a.e. in $J$ such

$$
\begin{aligned}
\text { that } x(0)= & 0, \alpha x(T)=\sum_{i=1}^{m} \beta_{i} I_{\eta_{i}}^{\gamma_{i}, \delta_{i}} x\left(\xi_{i}\right) \text { and } \\
& x(t)=J^{q} v(t)-\frac{t^{q-1}}{\Lambda}\left(\alpha J^{q} v(T)-\sum_{i=1}^{m} \beta_{i} I_{\eta_{i}}^{\gamma_{i}, \delta_{i}} J^{q} v\left(\xi_{i}\right)\right) .
\end{aligned}
$$

\section{Existence result via endpoint theory}

In this subsection we prove the existence of solutions for the problem (1) via endpoint theory.

Definition 3.1. An element $x \in X$ is called an endpoint of a multifunction $F: X \rightarrow \mathcal{P}(X)$ whenever $F x=\{x\}$ ([18]). Also, we say that $F$ has an approximate endpoint property whenever $\inf _{x \in X} \sup _{y \in F x} d(x, y)=0$ ([18]).

Definition 3.2. A function $f: \mathbb{R} \rightarrow \mathbb{R}$ is called upper semi-continuous whenever $\lim \sup _{n \rightarrow \infty} f\left(\lambda_{n}\right) \leq f(\lambda)$ for all sequence $\left\{\lambda_{n}\right\}_{n \geq 1}$ with $\lambda_{n} \rightarrow \lambda$.

For the proof of our first result, we need the following endpoint fixed point theorem $([18])$. 
Lemma 3.1. Let $\psi:[0, \infty) \rightarrow[0, \infty)$ be an upper semi-continuous function such that $\psi(t)<t$ and $\liminf _{t \rightarrow \infty}(t-\psi(t))>0$ for all $t>0$. Let $(X, d)$ be a complete metric space and $\mathcal{P}_{c l, b d}(X)$ is the collection of all nonempty closed and bounded subsets of $X$. Further $S: X \rightarrow \mathcal{P}_{c l, b d}(X)$ is a multifunction such that

$$
H_{d}(S x, S y) \leq \psi(d(x, y)) \text { for all } x, y \in X .
$$

Then $S$ has a unique endpoint if and only if $S$ has approximate endpoint property.

Based on Lemma 2.2, we introduce an operator $\mathcal{G}: \mathcal{U} \rightarrow \mathcal{P}(\mathcal{U})$ as follows:

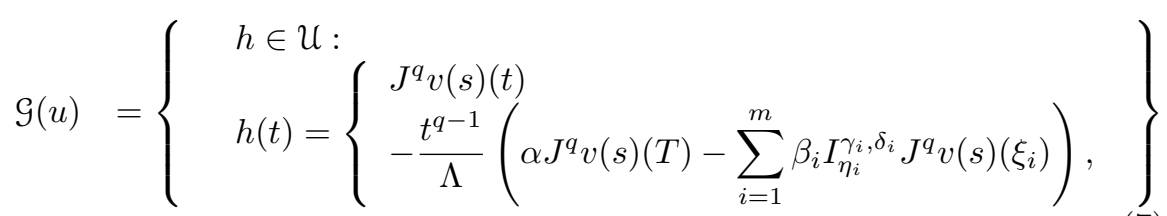

for $v \in S_{F, u}$, where $S_{F, u}$ denote the set of selections of $F$ defined by

$$
S_{F, u}:=\left\{v \in L^{1}(J, \mathbb{R}): v(t) \in F(t, u(t)) \text { for a.e. } t \in J\right\} .
$$

Now we are in a position to present our main existence result.

Theorem 3.1. Suppose that $\psi:[0, \infty) \rightarrow[0, \infty)$ is a nondecreasing upper semi-continuous mapping such that $\liminf _{t \rightarrow \infty}(t-\psi(t))>0$ and $\psi(t)<t$ for all $t>0$. Also, let $F: J \times \mathbb{R} \rightarrow \mathcal{P}_{c p}(\mathbb{R})$ be an integrable bounded multifunction such that $F(\cdot, u): J \rightarrow \mathcal{P}_{c p}(\mathbb{R})$ is measurable for all $u \in \mathbb{R}$, where $\mathcal{P}_{c p}(\mathbb{R})$ is the collection of all nonempty compact subsets of $\mathbb{R}$. Assume that there exists a function $\ell \in C(J,[0, \infty))$ such that

$$
H_{d}(F(t, u(t))-F(t, v(t))) \leq(\|\ell\| \theta)^{-1} \ell(t) \psi(|u(t)-v(t)|),
$$

where

$$
\theta=\frac{T^{q}}{\Gamma(q+1)}+\frac{|\alpha| T^{2 q-1}}{|\Lambda| \Gamma(q+1)}+\frac{T^{q-1}}{|\Lambda| \Gamma(q+1)} \sum_{i=1}^{m} \frac{\left|\beta_{i}\right| \xi_{i}^{q} \Gamma\left(\gamma_{i}+\left(q / \eta_{i}\right)+1\right)}{\Gamma\left(\gamma_{i}+\left(q / \eta_{i}\right)+\delta_{i}+1\right)} .
$$

If the multifunction $\mathcal{G}$ has the approximate endpoint property, then the inclusion problem (1) has a solution.

Proof. Our proof will be complete when we establish that the multifunction $\mathcal{G}: \mathcal{U} \rightarrow \mathcal{P}(\mathcal{U})$ defined by (7) has an endpoint. To do this, we show that the operator $\mathcal{G}(u)$ is a closed subset of $\mathcal{P}(\mathcal{U})$ for all $u \in \mathcal{U}$. Since the multivalued 
map $t \mapsto F(t, u(t))$ is measurable and has closed values for all $u \in \mathcal{U}$, so it has measurable selection. This implies that $S_{F, u}$ is nonempty for all $u \in \mathcal{U}$. Let $\left\{z_{n}\right\}_{n \geq 1}$ be a sequence in $\mathcal{G}(u)$ with $z_{n} \rightarrow z$ for $u \in \mathcal{U}$. For every $n \in \mathbb{N}$, choose $v_{n} \in S_{F, u_{n}}$ such that

$$
z_{n}(t)=J^{q} v_{n}(s)(t)-\frac{t^{q-1}}{\Lambda}\left(\alpha J^{q} v_{n}(s)(T)-\sum_{i=1}^{m} \beta_{i} I_{\eta_{i}}^{\gamma_{i}, \delta_{i}} J^{q} v_{n}(s)\left(\xi_{i}\right)\right) .
$$

By compactness of $F$, the sequence $\left\{v_{n}\right\}_{n \geq 1}$ has a subsequence which converges to some $v \in L^{1}(J)$. We denote this subsequence again by $\left\{v_{n}\right\}_{n \geq 1}$. It is clear that $v \in S_{F, u}$ and for all $t \in J$,

$$
z_{n}(t) \rightarrow z(t)=J^{q} v(s)(t)-\frac{t^{q-1}}{\Lambda}\left(\alpha J^{q} v(s)(T)-\sum_{i=1}^{m} \beta_{i} I_{\eta_{i}}^{\gamma_{i}, \delta_{i}} J^{q} v(s)\left(\xi_{i}\right)\right)
$$

This shows that $z \in \mathcal{G}(u)$ and so $\mathcal{G}$ is closed-valued. On the other hand, $\mathcal{G}(u)$ is a bounded set for all $u \in \mathcal{U}$, because $F$ is a compact multivalued map.

Finally, we show that $H_{d}(\mathcal{G}(u), \mathcal{G}(w)) \leq \psi(\|u-w\|)$. Let $u, w \in \mathcal{U}$ and $h_{1} \in \mathcal{G}(w)$. Choose $v_{1} \in S_{F, w}$ such that

$$
h_{1}(t)=J^{q} v_{1}(s)(t)-\frac{t^{q-1}}{\Lambda}\left(\alpha J^{q} v_{1}(s)(T)-\sum_{i=1}^{m} \beta_{i} I_{\eta_{i}}^{\gamma_{i}, \delta_{i}} J^{q} v_{1}(s)\left(\xi_{i}\right)\right),
$$

for almost all $t \in J$. Since

$$
H_{d}(F(t, u(t))-F(t, w(t))) \leq(\|\ell\| \theta)^{-1} \ell(t) \psi(|u(t)-w(t)|), \text { for all } t \in J,
$$

there exist $z \in F(t, u(t))$ provided that

$$
\left|v_{1}(t)-z\right| \leq(\|\ell\| \theta)^{-1} \ell(t) \psi(|u(t)-w(t)|), \text { for all } t \in J .
$$

Now, we consider the multivalued map $\mathcal{V}: J \rightarrow \mathcal{P}(\mathbb{R})$ given by

$$
\mathcal{V}(t)=\left\{z \in \mathbb{R}:\left|v_{1}(t)-z\right| \leq(\|\ell\| \theta)^{-1} \ell(t) \psi(|u(t)-w(t)|)\right\} .
$$

Since $v_{1}$ and $\varphi=(\|\ell\| \theta)^{-1} \ell \psi(|u-w|)$ are measurable, the multifunction $\mathcal{V}(\cdot) \cap F(\cdot, u(\cdot))$ is measurable. Choose $v_{2}(t) \in F(t, u(t))$ such that

$$
\left|v_{1}(t)-v_{2}(t)\right| \leq(\|\ell\| \theta)^{-1} \ell(t) \psi(|u(t)-w(t)|), \text { for all } t \in J
$$


We define the element $h_{2} \in \mathcal{G}(u)$ as follows:

$h_{2}(t)=J^{q} v_{2}(s)(t)-\frac{t^{q-1}}{\Lambda}\left(\alpha J^{q} v_{2}(s)(T)-\sum_{i=1}^{m} \beta_{i} I_{\eta_{i}}^{\gamma_{i}, \delta_{i}} J^{q} v_{2}(s)\left(\xi_{i}\right)\right)$, for all $t \in J$.

Let $\sup _{t \in J}|\ell(t)|=\|\ell\|$. Then, one can get

$$
\begin{aligned}
& \left|h_{1}(t)-h_{2}(t)\right| \\
\leq \quad & J^{q}\left|v_{1}(s)-v_{2}(s)\right|(t) \\
& +\frac{t^{q-1}}{|\Lambda|}\left(\alpha J^{q}\left|v_{1}(s)-v_{2}(s)\right|(T)+\sum_{i=1}^{m} \beta_{i} I_{\eta_{i}}^{\gamma_{i}, \delta_{i}} J^{q}\left|v_{1}(s)-v_{2}(s)\right|\left(\xi_{i}\right)\right) \\
\leq & (\|\ell\| \theta)^{-1}(\|\ell\| \theta) \psi(\|u-w\|) \\
= & \psi(\|u-w\|) .
\end{aligned}
$$

Hence, $\left\|h_{1}-h_{2}\right\| \leq \psi(\|u-w\|)$. Therefore $H_{d}(\mathcal{G}(u), \mathcal{G}(w)) \leq \psi(\|u-w\|)$ for all $u, w \in \mathcal{U}$. By hypothesis, since the multifunction $\mathcal{G}$ has approximate endpoint property, by Lemma 3.1, there exists $u^{*} \in \mathcal{U}$ such that $\mathcal{G}\left(u^{*}\right)=$ $\left\{u^{*}\right\}$. Consequently, the problem (1) has the solution $u^{*}$.This completes the proof.

Example 3.1. Consider the following boundary value problem for fractional differential inclusion with Erdélyi-Kober fractional integral conditions

$$
\left\{\begin{array}{l}
D^{3 / 2} x(t) \in F(t, x(t)), \quad t \in[0,5], \\
x(0)=0, \quad \frac{2}{3} x(5)=\frac{e}{2} I_{\frac{\sqrt{3}}{5}}^{\frac{5}{3}, \frac{3}{7}} x\left(\frac{4}{3}\right)+\frac{\pi}{3} I_{\frac{\sqrt{2}}{5}}^{\frac{2}{9}} \frac{\sqrt{3}}{8} x\left(\frac{3}{2}\right)+\frac{\sqrt{\pi}}{6} I_{\frac{e}{3}}^{\frac{\sqrt{e}}{2}}, \frac{e^{2}}{4} x\left(\frac{2}{7}\right) .
\end{array}\right.
$$

Here $q=3 / 2, m=3, T=5, \alpha=2 / 3, \beta_{1}=e / 2, \beta_{2}=\pi / 3, \beta_{3}=\sqrt{\pi} / 6$, $\eta_{1}=\sqrt{3} / 5, \eta_{2}=\sqrt{2} / 5, \eta_{3}=e / 3, \gamma_{1}=5 / 3, \gamma_{2}=2 / 9, \gamma_{3}=\sqrt{e} / 2, \delta_{1}=3 / 7$, $\delta_{2}=\sqrt{3} / 8, \delta_{3}=e^{2} / 4, \xi_{1}=4 / 3, \xi_{2}=3 / 2, \xi_{3}=2 / 7$. Using the given data, we find that

$\theta=\frac{T^{q}}{\Gamma(q+1)}+\frac{|\alpha| T^{2 q-1}}{|\Lambda| \Gamma(q+1)}+\frac{T^{q-1}}{|\Lambda| \Gamma(q+1)} \sum_{i=1}^{m} \frac{\left|\beta_{i}\right| \xi_{i}^{q} \Gamma\left(\gamma_{i}+\left(q / \eta_{i}\right)+1\right)}{\Gamma\left(\gamma_{i}+\left(q / \eta_{i}\right)+\delta_{i}+1\right)} \approx 44.000$

Let $F:[0,5] \times \mathbb{R} \rightarrow \mathcal{P}(\mathbb{R})$ be a multi-valued map given by

$$
x \rightarrow F(t, x)=\left[0, \frac{3 t}{10} \frac{|x|}{1+|x|}+\frac{2}{3}\right] .
$$


Setting $\ell(t)=(3 t) / 10, t \in[0,5]$, we have $\|\ell\|=3 / 2$. Choosing $\psi(y)=y / 2$, it is clear the function $\psi$ is nondecreasing upper semi-continuous on $[0,5]$ such that $\liminf _{y \rightarrow \infty}(y-\psi(y))>0$ and $\psi(y)<y$ for all $y>0$. Also

$$
H_{d}(F(t, x)-F(t, \bar{x})) \leq \frac{3 t}{10}|x-\bar{x}|<(\|\ell\| \theta)^{-1} \frac{3 t}{10} \psi(|x-\bar{x}|),
$$

for $x, \bar{x} \in \mathbb{R}$. Let $\mathcal{U}=C([0,5], \mathbb{R})$. We define an operator $\mathcal{G}: \mathcal{U} \rightarrow \mathcal{P}(\mathcal{U})$ by $\mathcal{G}(u)=\left\{z \in \mathcal{U}:\right.$ there exists $v \in S_{F, u}$ such that $z(t)=w(t)$ for all $\left.t \in[0,5]\right\}$,

where

$w(t)=J^{q} v(s)(t)-\frac{t^{q-1}}{\Lambda}\left(\alpha J^{q} v(s)(T)-\sum_{i=1}^{m} \beta_{i} I_{\eta_{i}}^{\gamma_{i}, \delta_{i}} J^{q} v(s)\left(\xi_{i}\right)\right), t \in[0,5]$.

Since $\sup _{u \in \mathcal{G}(0)}\|u\|=0$, thus $\inf _{u \in \mathcal{U}} \sup _{s \in \mathcal{G}(u)}\|u-s\|=0$. Consequently, the operator $\mathcal{G}$ has the approximate endpoint property. Thus all the conditions of Theorem 3.1 are satisfied. Therefore, by the conclusion of Theorem 3.1, the problem (9) with $F(t, x)$ given by (10) has at least one solution on $[0,5]$.

\section{Existence result via Krasnoselskii's multi-valued fixed point theorem}

In this subsection we prove the existence of solutions for the problem (1) with a convex valued right hand side, by applying Krasnoselskii's multi-valued fixed point theorem [19] and assuming that $F$ is $L^{1}$-Carathéodory.

Definition 4.1. A multivalued map $F:[0, T] \times \mathbb{R} \rightarrow \mathcal{P}(\mathbb{R})$ is said to be Carathéodory if (i) $t \longmapsto F(t, x)$ is measurable for each $x \in \mathbb{R}$ and (ii) $x \longmapsto F(t, x)$ is upper semicontinuous for almost all $t \in[0, T]$. Further $a$ Carathéodory function $F$ is called $L^{1}$-Carathéodory if (iii) for each $\rho>0$, there exists $\varphi_{\rho} \in L^{1}\left([0, T], \mathbb{R}^{+}\right)$such that $\|F(t, x)\|=\sup \{|v|: v \in F(t, x)\} \leq$ $\varphi_{\rho}(t)$ for all $\|x\| \leq \rho$ and for a.e. $t \in[0, T]$.

Lemma 4.1. ([15, Proposition 1.2]) If $G: X \rightarrow \mathcal{P}_{c l}(Y)$ is u.s.c., then $\operatorname{Gr}(G)$ is a closed subset of $X \times Y$; i.e., for every sequence $\left\{x_{n}\right\}_{n \in \mathbb{N}} \subset X$ and $\left\{y_{n}\right\}_{n \in \mathbb{N}} \subset Y$, if when $n \rightarrow \infty, x_{n} \rightarrow x_{*}, y_{n} \rightarrow y_{*}$ and $y_{n} \in G\left(x_{n}\right)$, then $y_{*} \in G\left(x_{*}\right)$. Conversely, if $G$ is completely continuous and has a closed graph, then it is upper semi-continuous.

Lemma 4.2. ([20]) Let $X$ be a Banach space. Let $F:[0, T] \times \mathbb{R} \rightarrow \mathcal{P}_{c p, c}(X)$ be an $L^{1}$ - Carathéodory multivalued map and let $\Theta$ be a linear continuous mapping from $L^{1}([0, T], X)$ to $C([0, T], X)$. Then the operator

$\Theta \circ S_{F}: C([0, T], X) \rightarrow \mathcal{P}_{c p, c}(C([0, T], X)), \quad x \mapsto\left(\Theta \circ S_{F}\right)(x)=\Theta\left(S_{F, x, y}\right)$ 
is a closed graph operator in $C([0, T], X) \times C([0, T], X)$.

Lemma 4.3. (Krasnoselskii's fixed point theorem [19]) Let $X$ be a Banach space, $Y \in \mathcal{P}_{b, c l, c}(X)$ and $A, B: Y \rightarrow \mathcal{P}_{c p, c}(X)$ are two multivalued operators. Then there exists $y \in Y$ such that $y \in A y+B y$ provided the operators $A$ and $B$ satisfy the conditions: (i) $A y+B y \subset Y$ for all $y \in Y$; (ii) $A$ is contraction; and (iii) $B$ is u.s.c and compact.

Theorem 4.1. Assume that:

$\left(H_{1}\right) F:[0, T] \times \mathbb{R} \rightarrow \mathcal{P}_{c p, c}(\mathbb{R})$ is $L^{1}$-Carathéodory multivalued map;

$\left(H_{2}\right)$ there exists a continuous function $p \in C\left([0, T], \mathbb{R}^{+}\right)$such that

$$
\|F(t, x)\|_{\mathcal{P}}:=\sup \{|y|: y \in F(t, x)\} \leq p(t), \quad \text { for each }(t, x) \in[0, T] \times \mathbb{R}
$$

$\left(H_{3}\right)$ there exists a function $k \in \mathcal{U}$ such that

$$
H_{d}(F(t, x), F(t, y)) \leq\|k\|\|x-y\|
$$

for a.e $t \in[0, T]$ and all $x, y \in \mathcal{U}$, with

$$
\|k\| \frac{T^{q-1}}{|\Lambda| \Gamma(q+1)} \sum_{i=1}^{m} \frac{\left|\beta_{i}\right| \xi_{i}^{q} \Gamma\left(\gamma_{i}+\left(q / \eta_{i}\right)+1\right)}{\Gamma\left(\gamma_{i}+\left(q / \eta_{i}\right)+\delta_{i}+1\right)}<1 .
$$

Then the boundary value problem (1) has at least one solution on $[0, T]$.

Proof. Let us introduce the multi-valued operators $\mathcal{A}: \mathcal{U} \longrightarrow \mathcal{P}(\mathcal{U})$ by

$$
\mathcal{A}(x)=\left\{h \in \mathcal{U}: h(t)=\frac{t^{q-1}}{\Lambda} \sum_{i=1}^{m} \beta_{i} I_{\eta_{i}}^{\gamma_{i}, \delta_{i}} J^{q} f(s)\left(\xi_{i}\right)\right\},
$$

and $\mathcal{B}: \mathcal{U} \longrightarrow \mathcal{P}(\mathcal{U})$ by

$$
\mathcal{B}(x)=\left\{h \in \mathcal{U}: h(t)=J^{\alpha} f(s)(t)-\alpha \frac{t^{q-1}}{\Lambda} J^{q} f(s)(T)\right\} .
$$

Observe that $\mathcal{G}=\mathcal{A}+\mathcal{B}$, where the operator $\mathcal{G}: \mathcal{U} \longrightarrow \mathcal{P}(\mathcal{U})$ is given by (7). We shall show that the operators $\mathcal{A}$ and $\mathcal{B}$ satisfy the conditions of Lemma 4.3 on $[0, T]$ in several steps. We begin by showing that the operators $\mathcal{A}$ and $\mathcal{B}$ define the multivalued operators $\mathcal{A}, \mathcal{B}: B_{r} \rightarrow \mathcal{P}_{c p, c}(\mathcal{U})$ where $B_{r}=\{x \in \mathcal{U}:\|x\| \leq r\}$ is a bounded set in $\mathcal{U}$. We will only prove that the operator $\mathcal{B}$ is compactvalued on $B_{r}$ and convex for all $x \in \mathcal{U}$ as the similar steps work for the operator 
$\mathcal{A}$. Note that the operator $\mathcal{B}$ is equivalent to the composition $\mathcal{L} \circ S_{F}$, where $\mathcal{L}$ is the continuous linear operator on $L^{1}([0, T], \mathbb{R})$ into $\mathcal{U}$, defined by

$$
\mathcal{L}(v)(t)=J^{\alpha} v(s)(t)-\alpha \frac{t^{q-1}}{\Lambda} J^{q} v(s)(T) .
$$

Suppose that $x \in B_{r}$ is arbitrary and let $\left\{v_{n}\right\}$ be a sequence in $S_{F, x}$. Then, by definition of $S_{F, x}$, we have $v_{n}(t) \in F(t, x(t))$ for almost all $t \in[0, T]$. Since $F(t, x(t))$ is compact for all $t \in J$, there is a convergent subsequence of $\left\{v_{n}(t)\right\}$ (we denote it by $\left\{v_{n}(t)\right\}$ again) that converges in measure to some $v(t) \in S_{F, x}$ for almost all $t \in J$. On the other hand, $\mathcal{L}$ is continuous, so $\mathcal{L}\left(v_{n}\right)(t) \rightarrow \mathcal{L}(v)(t)$ pointwise on $[0, T]$.

In order to show that the convergence is uniform, we have to show that $\left\{\mathcal{L}\left(v_{n}\right)\right\}$ is an equi-continuous sequence. Let $t_{1}, t_{2} \in[0, T]$ with $t_{1}<t_{2}$. Then, we have

$$
\begin{aligned}
& \left|\mathcal{L}\left(v_{n}\right)\left(t_{2}\right)-\mathcal{L}\left(v_{n}\right)\left(t_{1}\right)\right| \\
\leq & \left|J^{\alpha} v_{n}(s)\left(t_{2}\right)-J^{\alpha} v_{n}(s)\left(t_{1}\right)\right|+|\alpha| \frac{\left|t_{2}^{q-1}-t_{1}^{q-1}\right|}{|\Lambda|} J^{q}\left|v_{n}(s)\right|(T) \\
\leq & \frac{1}{\Gamma(q)}\left|\int_{0}^{t_{1}}\left[\left(t_{2}-s\right)^{q-1}-\left(t_{1}-s\right)^{q-1}\right] p(s) d s+\int_{t_{1}}^{t_{2}}\left(t_{2}-s\right)^{q-1} p(s) d s\right| \\
& +|\alpha| \frac{t_{2}^{q-1}-t_{1}^{q-1} \mid}{|\Lambda|} J^{q}\left|v_{n}(s)\right|(T) \\
\leq & \frac{\|p\|}{\Gamma(q+1)}\left[t_{2}^{q}-t_{1}^{q}\right]+|\alpha|\|p\| \frac{\left|t_{2}^{q-1}-t_{1}^{q-1}\right|}{|\Lambda|} \frac{T^{q}}{\Gamma(q+1)} .
\end{aligned}
$$

We see that the right hand of the above inequality tends to zero as $t_{2} \rightarrow t_{1}$. Thus, the sequence $\left\{\mathcal{L}\left(v_{n}\right)\right\}$ is equi-continuous and hence it follows by the Arzelá-Ascoli theorem that there exists a uniformly convergent subsequence $\left\{v_{n}\right\}$ (we denote it again by $\left\{v_{n}\right\}$ ) such that $\mathcal{L}\left(v_{n}\right) \rightarrow \mathcal{L}(v)$. Note that $\mathcal{L}(v) \in$ $\mathcal{L}\left(S_{F, x}\right)$. Hence, $\mathcal{B}(x)=\mathcal{L}\left(S_{F, x}\right)$ is compact for all $x \in B_{r}$. So $\mathcal{B}(x)$ is compact.

To establish that $\mathcal{B}(x)$ is convex for all $x \in \mathcal{U}$. Let $z_{1}, z_{2} \in \mathcal{B}(x)$. We select $f_{1}, f_{2} \in S_{F, x}$ such that

$$
z_{i}(t)=J^{\alpha} f_{i}(s)(t)-\alpha \frac{t^{q-1}}{\Lambda} J^{q} f_{i}(s)(T), \quad i=1,2,
$$

for almost all $t \in[0, T]$. Let $0 \leq \lambda \leq 1$. Then, we have

$$
\left[\lambda z_{1}+(1-\lambda) z_{2}\right](t)=J^{\alpha}\left[\lambda f_{1}(s)+(1-\lambda) f_{2}(s)\right](t)
$$




$$
-\alpha \frac{t^{q-1}}{\Lambda} J^{q}\left[\lambda f_{1}(s)+(1-\lambda) f_{2}(s)\right](T) .
$$

Since $F$ has convex values, so $S_{F, u}$ is convex and $\lambda f_{1}(s)+(1-\lambda) f_{2}(s) \in S_{F, x}$. Thus $\lambda z_{1}+(1-\lambda) z_{2} \in \mathcal{B}(x)$. Consequently, $\mathcal{B}$ is convex-valued.

The rest of the proof consists of the following steps and claims.

Step 1: We show that $\mathcal{A}$ is a multi-valued contraction on $\mathcal{U}$. Let $x, y \in \mathcal{U}$ and $h_{1} \in \mathcal{A} x$. Then there exists $v_{1}(t) \in F(t, x(t))$ such that, for each $t \in[0, T]$,

$$
h_{1}(t)=\frac{t^{q-1}}{\Lambda} \sum_{i=1}^{m} \beta_{i} I_{\eta_{i}}^{\gamma_{i}, \delta_{i}} J^{q} v_{1}(s)\left(\xi_{i}\right) .
$$

Since $H_{d}(F(t, x), F(t, y)) \leq k(t)\|x-y\|$, there exists $w \in F(t, y)$ such that

$$
\left|v_{1}(t)-w\right| \leq k(t)\|x-y\|
$$

Thus the multi-valued operator $U$ defined by $U(t)=S_{F, y} \cap K(t)$, where

$$
K(t)=\left\{w \in \mathbb{R}:\left|v_{1}(t)-w\right| \leq k(t)\|x-y\|\right\},
$$

is nonempty and measurable. Let $v_{2}$ be a measurable selection for $U$ (which exists by Kuratowski-Ryll-Nardzewski's selection theorem [16]). Then $v_{2}(t) \in$ $F(t, y(t))$ and for each $t \in[0, T]$, we have $\left|v_{1}(t)-v_{2}(t)\right| \leq k(t)\|x-y\|$ a.e. on $[0, T]$.

For each $t \in[0, T]$, let us define

$$
h_{2}(t)=\frac{t^{q-1}}{\Lambda} \sum_{i=1}^{m} \beta_{i} I_{\eta_{i}}^{\gamma_{i}, \delta_{i}} J^{q} v_{2}(s)\left(\xi_{i}\right) .
$$

It follows that $h_{2} \in \mathcal{A} y$ and

$$
\begin{aligned}
\left|h_{1}(t)-h_{2}(t)\right| & \leq \frac{t^{q-1}}{|\Lambda|} \sum_{i=1}^{m} \beta_{i} I_{\eta_{i}}^{\gamma_{i}, \delta_{i}} J^{q}\left|v_{1}(s)-v_{2}(s)\right|\left(\xi_{i}\right) \\
& \leq\|k\| \frac{T^{q-1}}{|\Lambda| \Gamma(q+1)} \sum_{i=1}^{m} \frac{\left|\beta_{i}\right| \xi_{i}^{q} \Gamma\left(\gamma_{i}+\left(q / \eta_{i}\right)+1\right)}{\Gamma\left(\gamma_{i}+\left(q / \eta_{i}\right)+\delta_{i}+1\right)}\|x-y\| .
\end{aligned}
$$

Taking supremum over $t \in[0, T]$, we obtain

$$
\left\|h_{1}-h_{2}\right\| \leq\|k\| \frac{T^{q-1}}{|\Lambda| \Gamma(q+1)} \sum_{i=1}^{m} \frac{\left|\beta_{i}\right| \xi_{i}^{q} \Gamma\left(\gamma_{i}+\left(q / \eta_{i}\right)+1\right)}{\Gamma\left(\gamma_{i}+\left(q / \eta_{i}\right)+\delta_{i}+1\right)}\|x-y\|,
$$


which together with the analogous inequality obtained by interchanging the roles of $x$ and $y$ yields

$$
H_{d}(\mathcal{A} x, \mathcal{A} y) \leq\|k\| \frac{T^{q-1}}{|\Lambda| \Gamma(q+1)} \sum_{i=1}^{m} \frac{\left|\beta_{i}\right| \xi_{i}^{q} \Gamma\left(\gamma_{i}+\left(q / \eta_{i}\right)+1\right)}{\Gamma\left(\gamma_{i}+\left(q / \eta_{i}\right)+\delta_{i}+1\right)}\|x-y\|,
$$

for each $x, y \in \mathcal{U}$. This shows that $\mathcal{A}$ is a multi-valued contraction, since

$$
\|k\| \frac{T^{q-1}}{|\Lambda| \Gamma(q+1)} \sum_{i=1}^{m} \frac{\left|\beta_{i}\right| \xi_{i}^{q} \Gamma\left(\gamma_{i}+\left(q / \eta_{i}\right)+1\right)}{\Gamma\left(\gamma_{i}+\left(q / \eta_{i}\right)+\delta_{i}+1\right)}<1 .
$$

Step 2: $\mathcal{B}$ is compact and upper semicontinuous. This will be established in several claims.

Claim I: $\mathcal{B}$ maps bounded sets into bounded sets in $\mathcal{U}$.

For a positive number $\rho$, let $B_{\rho}=\{x \in \mathcal{U}:\|x\| \leq \rho\}$ be a bounded ball in $\mathcal{U}$. Then, for each $h \in \mathcal{G}(x), x \in B_{\rho}$, there exists $v \in S_{F, x}$ such that

$$
h(t)=J^{q} v(s)(t)-\frac{t^{q-1}}{\Lambda} \alpha J^{q} v(s)(T) .
$$

Then we have

$$
\begin{aligned}
|h(x)| & \leq J^{q}|v(s)|(T)+\frac{|\alpha| T^{q-1}}{|\Lambda|} J^{q}|v(s)|(T) \\
& \leq J^{q} p(s)(T)+\frac{|\alpha| T^{q-1}}{|\Lambda|} J^{q} p(s)(T) \\
& \leq\|p\|\left(\frac{T^{q}}{\Gamma(q+1)}+\frac{|\alpha| T^{2 q-1}}{|\Lambda| \Gamma(q+1)}\right) .
\end{aligned}
$$

and consequently,

$$
\|h\| \leq\|p\|\left(\frac{T^{q}}{\Gamma(q+1)}+\frac{|\alpha| T^{2 q-1}}{|\Lambda| \Gamma(q+1)}\right) .
$$

Claim II: $\mathcal{B}$ maps bounded sets into equi-continuous sets.

Let $\tau_{1}, \tau_{2} \in[0, T]$ with $\tau_{1}<\tau_{2}$ and $x \in B_{\rho}$. For each $h \in \mathcal{G}(x)$, we obtain

$$
\begin{aligned}
& \left|h\left(\tau_{2}\right)-h\left(\tau_{1}\right)\right| \\
\leq & \left|J^{q} v(s)\left(\tau_{2}\right)-J^{q} v(s)\left(\tau_{1}\right)\right|+\frac{|\alpha|\left|\tau_{2}^{q-1}-\tau_{1}^{q-1}\right|}{|\Lambda|} J^{q}|v(s)|(T)
\end{aligned}
$$




$$
\begin{aligned}
\leq & \frac{1}{\Gamma(q)}\left|\int_{0}^{\tau_{1}}\left[\left(\tau_{2}-s\right)^{q-1}-\left(\tau_{1}-s\right)^{q-1}\right] p(s) d s+\int_{\tau_{1}}^{\tau_{2}}\left(\tau_{2}-s\right)^{q-1} p(s) d s\right| \\
& +\frac{\left|\tau_{2}^{q-1}-\tau_{1}^{q-1}\right|}{|\Lambda|}|\alpha| J^{q} p(s)(T) \\
\leq & \frac{\|p\|}{\Gamma(q+1)}\left[t_{2}^{q}-t_{1}^{q}\right]+|\alpha|\|p\| \frac{\left|t_{2}^{q-1}-t_{1}^{q-1}\right|}{|\Lambda|} \frac{T^{q}}{\Gamma(q+1)} .
\end{aligned}
$$

Obviously the right hand side of the above inequality tends to zero independently of $x \in B_{r}$ as $\tau_{2}-\tau_{1} \rightarrow 0$. Therefore we deduce by the Ascoli-Arzelá theorem that $\mathcal{B}: \mathcal{U} \rightarrow \mathcal{P}(\mathcal{U})$ is completely continuous.

By Claims I and II, $\mathcal{B}$ is completely continuous. By Lemma $4.1, \mathcal{B}$ will be upper semicontinuous (since it is completely continuous) if we show that it has a closed graph. Thus, in the next claim, we prove it.

Claim III: $\mathcal{B}$ has a closed graph.

Let $x_{n} \rightarrow x_{*}, h_{n} \in \mathcal{B}\left(x_{n}\right)$ and $h_{n} \rightarrow h_{*}$. Then we need to show that $h_{*} \in \mathcal{B}\left(x_{*}\right)$. Associated with $h_{n} \in \mathcal{B}\left(x_{n}\right)$, there exists $f_{n} \in S_{F, x_{n}}$ such that for each $t \in[0, T]$,

$$
h(t)=J^{\alpha} f_{n}(s)(t)-\alpha \frac{t^{q-1}}{\Lambda} J^{q} f_{n}(s)(T) .
$$

Thus it suffices to show that there exists $f_{*} \in S_{F, x_{*}}$ such that for each $t \in[0, T]$,

$$
h_{*}(t)=J^{\alpha} f_{*}(s)(t)-\alpha \frac{t^{q-1}}{\Lambda} J^{\alpha} f_{*}(s)(T) .
$$

Let us consider the linear operator $\Theta: L^{1}([0, T], \mathbb{R}) \rightarrow \mathcal{U}$ given by

$$
f \mapsto \Theta(f)(t)=J^{\alpha} f(s)(t)-\alpha \frac{t^{q-1}}{\Lambda} J^{\alpha} f(s)(T) .
$$

Observe that

$$
\begin{aligned}
\left\|h_{n}(t)-h_{*}(t)\right\|= & \| J^{\alpha}\left(f_{n}(s)-f_{*}(s)\right)(t) \\
& \left.-\alpha \frac{t^{q-1}}{\Lambda} f_{*}(s)\right)(\xi)-J^{\alpha}\left(f_{n}(s)-f_{*}(s)\right)(T) \| \rightarrow 0,
\end{aligned}
$$

as $n \rightarrow \infty$.

Thus, it follows by Lemma 4.2 that $\Theta \circ S_{F}$ is a closed graph operator. Further, we have $h_{n}(t) \in \Theta\left(S_{F, x_{n}}\right)$. Since $x_{n} \rightarrow x_{*}$, we have that

$$
h_{*}(t)=J^{\alpha} f_{*}(s)(t)-\alpha \frac{t^{q-1}}{\Lambda} J^{\alpha} f_{*}(s)(T),
$$


for some $f_{*} \in S_{F, x_{*}}$. Hence $\mathcal{B}$ has a closed graph (and therefore has closed values). In consequence, the operator $\mathcal{B}$ is compact and upper semicontinuous.

Step 3: Here, we show that $\mathcal{A}(x)+\mathcal{B}(x) \subset B_{r}$ for all $x \in B_{r}$. Suppose $x \in B_{r}$, with $r>\|p\| \theta(\theta$ defined by $(8))$ and $h \in \mathcal{B}$ are arbitrary elements. Choose $f \in S_{F, x}$ such that

$h(t)=J^{\alpha} f(s)(t)-\frac{t^{q-1}}{\Lambda}\left(\alpha J^{q} f(s)(T)-\sum_{i=1}^{m} \beta_{i} I_{\eta_{i}}^{\gamma_{i}, \delta_{i}} J^{q} f(s)\left(\xi_{i}\right)\right), t \in[0, T]$.

Then we have

$$
\begin{aligned}
|h(x)| & \leq J^{q}|f(s)|(T)+\frac{|\alpha| T^{q-1}}{|\Lambda|} J^{q}|f(s)|(T)+\frac{T^{q-1}}{|\Lambda|} \sum_{i=1}^{m}\left|\beta_{i}\right| I_{\eta_{i}}^{\gamma_{i}, \delta_{i}} J^{q}|f(s)|\left(\xi_{i}\right) \\
& \leq J^{q} p(s)(T)+\frac{|\alpha| T^{q-1}}{|\Lambda|} J^{q} p(s)(T)+\frac{T^{q-1}}{|\Lambda|} \sum_{i=1}^{m}\left|\beta_{i}\right| I_{\eta_{i}}^{\gamma_{i}, \delta_{i}} J^{q} p(s)\left(\xi_{i}\right) \\
& \leq\|p\|\left(\frac{T^{q}}{\Gamma(q+1)}+\frac{|\alpha| T^{2 q-1}}{|\Lambda| \Gamma(q+1)}+\frac{T^{q-1}}{|\Lambda| \Gamma(q+1)} \sum_{i=1}^{m} \frac{\left|\beta_{i}\right| \xi_{i}^{q} \Gamma\left(\gamma_{i}+\left(q / \eta_{i}\right)+1\right)}{\Gamma\left(\gamma_{i}+\left(q / \eta_{i}\right)+\delta_{i}+1\right)}\right) .
\end{aligned}
$$

Thus

$$
\|h\| \leq\|p\| \theta<r .
$$

Hence $\|h\| \leq r$, which means that $\mathcal{A}(x)+\mathcal{B}(x) \subset B_{r}$ for all $x \in B_{r}$.

Thus, the operators $\mathcal{A}$ and $\mathcal{B}$ satisfy all the conditions of Lemma 4.3 and hence we conclude that $x \in \mathcal{A}(x)+\mathcal{B}(x)$ has a solution in $B_{r}$. Therefore problem (1) has a solution in $B_{r}$ and the proof is completed.

Example 4.1. Let us consider problem (9) with the multi-valued map $F$ : $[0,5] \times \mathbb{R} \rightarrow \mathcal{P}(\mathbb{R})$ given by

$$
x \rightarrow F(t, x)=\left[\frac{\cos t}{\sqrt{t+25}}, \frac{1}{10 \sqrt{\left(1+t^{2}\right)}} \tan ^{-1} x+\frac{\cos t}{\sqrt{t+25}}\right] .
$$

Clearly

$$
\|F(t, x)\|_{\mathcal{P}}:=\sup \{|y|: y \in F(t, x)\} \leq \frac{\pi}{20 \sqrt{\left(1+t^{2}\right)}}+\frac{\cos t}{\sqrt{t+25}}=p(t),
$$

and $H_{d}(F(t, x), F(t, y)) \leq \frac{1}{10}|x-y|$, for $x, y \in \mathbb{R}$. With $\|k\|=1 / 10$, we find that

$$
\|k\| \frac{T^{q-1}}{|\Lambda| \Gamma(q+1)} \sum_{i=1}^{m} \frac{\Gamma\left(\gamma_{i}+\left(q / \eta_{i}\right)+1\right)}{\Gamma\left(\gamma_{i}+\left(q / \eta_{i}\right)+\delta_{i}+1\right)} \approx 0.818538<1 .
$$

Thus the hypotheses of Theorem 4.1 hold and consequently, problem (9) with $F(t, x)$ given by 14 has a solution by Theorem 4.1 . 


\subsection{Existence result via Wegrzyk's fixed point theorem}

In this subsection we prove the existence of solutions for problem (1) with a not necessary nonconvex valued right hand side, by applying Wegrzyk's fixed point theorem [21].

Definition 4.2. A function $\delta: \mathbb{R}_{+} \rightarrow \mathbb{R}_{+}$is said to be a strict comparison function (see [22]) if it is continuous, strictly increasing and $\sum_{n=1}^{\infty} \delta^{n}(t)<\infty$, for each $t>0$.

Definition 4.3. A multivalued operator $N: X \rightarrow \mathcal{P}_{c l}(X)$ is called (a) $\gamma$-Lipschitz if and only if there exists $\gamma>0$ such that $H_{d}(N(x), N(y)) \leq$ $\gamma d(x, y)$ for each $x, y \in X ;(b)$ a contraction if and only if it is $\gamma$-Lipschitz with $\gamma<1 ;(c)$ a generalized contraction if and only if there is a strict comparison function $\delta: \mathbb{R}_{+} \rightarrow \mathbb{R}_{+}$such that $H_{d}(N(x), N(y)) \leq \delta(d(x, y))$ for each $x, y \in$ $X$.

Lemma 4.4. (Wegrzyk's fixed point theorem [21]). Let $(X, d)$ be a complete metric space. If $\mathcal{Q}: X \rightarrow P_{c l}(X)$ is a generalized contraction, then Fix $\mathcal{Q} \neq \emptyset$.

Theorem 4.2. Assume that

$\left(A_{1}\right) F:[0, T] \times \mathbb{R} \rightarrow \mathcal{P}_{c p}(\mathbb{R})$ is such that $F(\cdot, x):[0, T] \rightarrow \mathcal{P}_{c p}(\mathbb{R})$ is measurable for each $x \in \mathbb{R}$.

$\left(A_{2}\right) H_{d}(F(t, x), F(t, \bar{x})) \leq m(t) \delta(|x-\bar{x}|)$ for almost all $t \in[0, T]$ and $x, \bar{x} \in \mathbb{R}$ with $m \in C\left([0, T], \mathbb{R}^{+}\right)$and $d(0, F(t, 0)) \leq m(t)$ for almost all $t \in[0, T]$, where $\delta: \mathbb{R}_{+} \rightarrow \mathbb{R}_{+}$is strictly increasing.

Then the boundary value problem (1) has at least one solution on $[0, T]$ if $\varepsilon \delta: \mathbb{R}_{+} \rightarrow \mathbb{R}_{+}$is a strict comparison function, where $\varepsilon=\|m\| \theta$, i.e.

$\varepsilon=\|m\|\left\{\frac{T^{q}}{\Gamma(q+1)}+\frac{|\alpha| T^{2 q-1}}{|\Lambda| \Gamma(q+1)}+\frac{T^{q-1}}{|\Lambda| \Gamma(q+1)} \sum_{i=1}^{m} \frac{\left|\beta_{i}\right| \xi_{i}^{q} \Gamma\left(\gamma_{i}+\left(q / \eta_{i}\right)+1\right)}{\Gamma\left(\gamma_{i}+\left(q / \eta_{i}\right)+\delta_{i}+1\right)}\right\}$.

Proof. Let $\varepsilon \delta: \mathbb{R}_{+} \rightarrow \mathbb{R}_{+}$be a strict comparison function. It follows by the assumptions $\left(A_{1}\right)$ and $\left(A_{2}\right)$ that $F(\cdot, x(\cdot))$ is measurable and has a measurable selection $v(\cdot)$ (see Theorem III.6 [23]). Also $m \in C([0,1], \mathbb{R})$ and

$$
\begin{aligned}
|v(t)| & \leq d(0, F(t, 0))+H_{d}(F(t, 0), F(t, x(t))) \\
& \leq m(t)+m(t) \delta(|x(t)|) \\
& \leq(1+\delta(\|x\|) m(t) .
\end{aligned}
$$

Thus the set $S_{F, x}$ is nonempty for each $x \in \mathcal{U}$. Now we show that the operator $\mathcal{G}$ satisfies the assumptions of Lemma 4.4. We show that $\mathcal{G}(x) \in \mathcal{P}_{c l}((\mathcal{U})$ for 
each $x \in \mathcal{U}$. Let $\left\{u_{n}\right\}_{n \geq 0} \in \mathcal{G}(x)$ be such that $u_{n} \rightarrow u$ in $\mathcal{U}$ as $n \rightarrow \infty$. Then $u \in \mathcal{U}$ and there exists $v_{n} \in S_{F, x_{n}}$ such that, for each $t \in[0, T]$,

$$
u_{n}(t)=J^{q} v_{n}(s)(t)-\frac{t^{q-1}}{\Lambda}\left(\alpha J^{q} v_{n}(s)(T)-\sum_{i=1}^{m} \beta_{i} I_{\eta_{i}}^{\gamma_{i}, \delta_{i}} J^{q} v_{n}(s)\left(\xi_{i}\right)\right) .
$$

As $F$ has compact values, we pass onto a subsequence (if necessary) to obtain that $v_{n}$ converges to $v$ in $L^{1}([0, T], \mathbb{R})$. Thus, $v \in S_{F, x}$ and for each $t \in[0, T]$, we have

$$
u_{n}(t) \rightarrow v(t)=J^{q} v(s)(t)-\frac{t^{q-1}}{\Lambda}\left(\alpha J^{q} v(s)(T)-\sum_{i=1}^{m} \beta_{i} I_{\eta_{i}}^{\gamma_{i}, \delta_{i}} J^{q} v(s)\left(\xi_{i}\right)\right) .
$$

Hence, $u \in \mathcal{G}(x)$.

Next we show that

$$
H_{d}(\mathcal{G}(x), \mathcal{G}(\bar{x})) \leq \varepsilon \delta(\|x-\bar{x}\|) \text { for each } x, \bar{x} \in \mathcal{U} .
$$

Let $x, \bar{x} \in \mathcal{U}$ and $h_{1} \in \mathcal{G}(x)$. Then there exists $v_{1}(t) \in F(t, x(t))$ such that, for each $t \in[0, T]$,

$$
h_{1}(t)=J^{q} v_{1}(s)(t)-\frac{t^{q-1}}{\Lambda}\left(\alpha J^{q} v_{1}(s)(T)-\sum_{i=1}^{m} \beta_{i} I_{\eta_{i}}^{\gamma_{i}, \delta_{i}} J^{q} v_{1}(s)\left(\xi_{i}\right)\right) .
$$

By $\left(A_{2}\right)$, we have

$$
H_{d}(F(t, x), F(t, \bar{x})) \leq m(t) \delta(|x(t)-\bar{x}(t)|) .
$$

So, there exists $w \in F(t, \bar{x}(t))$ such that

$$
\left|v_{1}(t)-w(t)\right| \leq m(t) \delta(|x(t)-\bar{x}(t)|), \quad t \in[0, T] .
$$

Define $U:[0, T] \rightarrow \mathcal{P}(\mathbb{R})$ by

$$
U(t)=\left\{w \in \mathbb{R}:\left|v_{1}(t)-w\right| \leq m(t) \delta(|x(t)-\bar{x}(t)|)\right\} .
$$

Since the multivalued operator $U(t) \cap F(t, \bar{x}(t)$ ) is measurable (Proposition III.4 [23]), there exists a function $v_{2}(t)$ which is a measurable selection for $U$. So $v_{2}(t) \in F(t, \bar{x}(t))$ and for each $t \in[0, T]$, we have $\left|v_{1}(t)-v_{2}(t)\right| \leq$ $m(t) \delta(|x(t)-\bar{x}(t)|)$.

For each $t \in[0, T]$, let us define

$$
h_{2}(t)=J^{q} v_{2}(s)(t)-\frac{t^{q-1}}{\Lambda}\left(\alpha J^{q} v_{2}(s)(T)-\sum_{i=1}^{m} \beta_{i} I_{\eta_{i}}^{\gamma_{i}, \delta_{i}} J^{q} v_{2}(s)\left(\xi_{i}\right)\right) .
$$


Thus,

$$
\begin{aligned}
& \left|h_{1}(t)-h_{2}(t)\right| \\
& \leq J^{q}\left|v_{1}(s)-v_{2}(s)\right|(t) \\
& +\frac{t^{q-1}}{|\Lambda|}\left(\alpha J^{q}\left|v_{1}(s)-v_{2}(s)\right|(T)+\sum_{i=1}^{m} \beta_{i} I_{\eta_{i}}^{\gamma_{i}, \delta_{i}} J^{q}\left|v_{1}(s)-v_{2}(s)\right|\left(\xi_{i}\right)\right) \\
& \leq\|m\|\left(\frac{T^{q}}{\Gamma(q+1)}+\frac{|\alpha| T^{2 q-1}}{|\Lambda| \Gamma(q+1)}\right. \\
& \left.+\frac{T^{q-1}}{|\Lambda| \Gamma(q+1)} \sum_{i=1}^{m} \frac{\left|\beta_{i}\right| \xi_{i}^{q} \Gamma\left(\gamma_{i}+\left(q / \eta_{i}\right)+1\right)}{\Gamma\left(\gamma_{i}+\left(q / \eta_{i}\right)+\delta_{i}+1\right)}\right) \delta(\|x-\bar{x}\|) .
\end{aligned}
$$

Hence,

$$
\begin{aligned}
\left\|h_{1}-h_{2}\right\| \leq & \|m\|\left(\frac{T^{q}}{\Gamma(q+1)}+\frac{|\alpha| T^{2 q-1}}{|\Lambda| \Gamma(q+1)}\right. \\
& \left.+\frac{T^{q-1}}{|\Lambda| \Gamma(q+1)} \sum_{i=1}^{m} \frac{\left|\beta_{i}\right| \xi_{i}^{q} \Gamma\left(\gamma_{i}+\left(q / \eta_{i}\right)+1\right)}{\Gamma\left(\gamma_{i}+\left(q / \eta_{i}\right)+\delta_{i}+1\right)}\right) \delta(\|x-\bar{x}\|) .
\end{aligned}
$$

Analogously, interchanging the roles of $x$ and $\bar{x}$, we obtain

$$
\begin{aligned}
& H_{d}(\mathcal{G}(x), \mathcal{G}(\bar{x})) \leq \varepsilon \delta(\|x-\bar{x}\|) \\
&=\|m\|\left(\frac{T^{q}}{\Gamma(q+1)}+\frac{|\alpha| T^{2 q-1}}{|\Lambda| \Gamma(q+1)}+\frac{T^{q-1}}{|\Lambda| \Gamma(q+1)} \sum_{i=1}^{m} \frac{\left|\beta_{i}\right| \xi_{i}^{q} \Gamma\left(\gamma_{i}+\left(q / \eta_{i}\right)+1\right)}{\Gamma\left(\gamma_{i}+\left(q / \eta_{i}\right)+\delta_{i}+1\right)}\right) .
\end{aligned}
$$

for each $x, \bar{x} \in \mathcal{U}$. Therefore, $\mathcal{G}$ is a generalized contraction. Thus it follows by Lemma 4.4 that $\mathcal{G}$ has a fixed point $x$ which is a solution of (1). This completes the proof.

Remark 4.1. Theorem 4.2 holds for several values of the function $\delta$, for example, $\delta(t)=\ln (1+t)^{3}, \delta(t)=t$, etc.

\section{References}

[1] M. Kisielewicz, Stochastic differential inclusions and applications, Springer Optimization and Its Applications, 80. Springer, New York, 2013. 
[2] B. Ahmad, S.K. Ntouyas, Existence results for higher order fractional differential inclusions with multi-strip fractional integral boundary conditions, Electron. J. Qual. Theory Differ. Equ. (2013), No. 20, 19 pp.

[3] S. Balochian, M. Nazari, Stability of particular class of fractional differential inclusion systems with input delay. Control Intell. Syst. 42 (2014), no. $4,279-283$.

[4] X. Wang, P. Schiavone, Harmonic three-phase circular inclusions in finite elasticity, Contin. Mech. Thermodyn. 27 (2015), no. 4-5, 739-747.

[5] J. Sun, Q. Yin, Robust fault-tolerant full-order and reduced-order observer synchronization for differential inclusion chaotic systems with unknown disturbances and parameters, J. Vib. Control 21 (2015), no. 11, 2134-2148.

[6] S.K. Ntouyas, S. Etemad, J. Tariboon, Existence results for multi-term fractional differential inclusions, Adv. Differ. Equ. 2015, 2015:140.

[7] B. Ahmad, R.P Agarwal, A. Alsaedi, Fractional differential equations and inclusions with semiperiodic and three-point boundary conditions, Bound. Value Probl. 2016, 2016:28, 20 pages.

[8] A. Erdélyi, H. Kober, Some remarks on Hankel transforms, Quart. J. Math., Oxford, Second Ser. 11 (1940), 212-221.

[9] I.N. Sneddon, The use in mathematical analysis of Erdélyi-Kober operators and some of their applications. In: Fractional Calculus and Its Applications, Proc. Internat. Conf. Held in New Haven, Lecture Notes in Math., 1975, 457, Springer, N. York, 37-79.

[10] S.L. Kalla, V.S. Kiryakova, An $H$-function generalized fractional calculus based upon compositions of Erdélyi-Kober operators in $L_{p}$, Math. Japonica 35 (1990), 1-21.

[11] S.B. Yakubovich, Yu.F. Luchko, The Hypergeometric Approach to Integral Transforms and Convolutions, Mathematics and its Appl. 287, Kluwer Acad. Publ., Dordrecht-Boston-London, 1994.

[12] V. Kiryakova, Generalized Fractional Calculus and Applications, Pitman Research Notes in Math. 301, Longman, Harlow - J. Wiley, N. York, 1994.

[13] A.A. Kilbas, H.M. Srivastava, J.J. Trujillo, Theory and Applications of Fractional Differential Equations, North-Holland Mathematics Studies, 204. Elsevier Science B.V., Amsterdam, 2006. 
[14] I. Podlubny, Fractional Differential Equations, Academic Press, San Diego, 1999.

[15] K. Deimling, Multivalued Differential Equations, Walter De Gruyter, Berlin-New York, 1992.

[16] Sh. Hu, N. Papageorgiou, Handbook of Multivalued Analysis, Theory I, Kluwer, Dordrecht, 1997.

[17] M. Kisielewicz, Differential Inclusions and Optimal Control, Kluwer, Dordrecht, The Netherlands, 1991.

[18] A. Amini-Harandi, Endpoints of set-valued contractions in metric spaces, Nonlinear Anal. 72 (2010) 132-134.

[19] A. Petrusel, Fixed points and selections for multivalued operators, Seminar on Fixed Point Theory Cluj-Napoca 2 ( 2001), 3-22.

[20] A. Lasota and Z. Opial, An application of the Kakutani-Ky Fan theorem in the theory of ordinary differential equations, Bull. Acad. Polon. Sci. Ser.Sci. Math. Astronom. Phys. 13 (1965), 781-786.

[21] R. Wegrzyk, Fixed point theorems for multifunctions and their applications to functional equations, Dissertationes Math. (Rozprawy Mat.) 201 (1982) 28.

[22] A. Granas, J. Dugundji, Fixed Point Theory, Springer-Verlag, New York, 2003.

[23] C. Castaing, M. Valadier, Convex Analysis and Measurable Multifunctions, Lecture Notes in Mathematics 580, Springer-Verlag, BerlinHeidelberg-New York, 1977.

Bashir AHMAD,

Nonlinear Analysis and Applied Mathematics (NAAM)-Research Group,

Department of Mathematics, Faculty of Science

King Abdulaziz University, P.O. Box 80203, Jeddah 21589, Saudi Arabia.

Email: bashirahmad_qau@yahoo.com

Sotiris K. NTOUYAS,

Department of Mathematics,

University of Ioannina,

45110 Ioannina, Greece.

Nonlinear Analysis and Applied Mathematics (NAAM)-Research Group,

Department of Mathematics, Faculty of Science

King Abdulaziz University, P.O. Box 80203, Jeddah 21589, Saudi Arabia.

Email: sntouyas@uoi.gr 